\title{
Prolonged static stretching causes acute, nonmetabolic fatigue and impairs exercise tolerance during severe-intensity cycling
}

\author{
Alessandro L. Colosio, Massimo Teso, and Silvia Pogliaghi
}

\begin{abstract}
We tested the hypothesis that static stretching, an acute, nonmetabolic fatiguing intervention, reduces exercise tolerance by increasing muscle activation and affecting muscle bioenergetics during cycling in the "severe" intensity domain. Ten active men (age, $24 \pm 2$ years; body mass, $74 \pm 11 \mathrm{~kg}$; height, $176 \pm 8 \mathrm{~cm}$ ) participated in identical constant-load cycling tests of equal intensity, of which 2 tests were carried out under control conditions and 2 were done after stretching. This resulted in a 5\% reduction of maximal isokinetic sprinting power output. We measured (i) oxygen consumption, (ii) electromyography, (iii) deoxyhemoglobin, (iv) blood lactate concentration; $(v)$ time to exhaustion, and (vi) perception of effort. Finally, oxygen consumption and deoxyhemoglobin kinetics were determined. Force reduction following stretching was accompanied by augmented muscle excitation at a given workload $(p=0.025)$ and a significant reduction in time to exhaustion $(p=0.002)$. The time to peak oxygen consumption was reduced by stretching $(p=0.034)$, suggesting an influence of the increased muscle excitation on the oxygen consumption kinetics. Moreover, stretching was associated with a mismatch between $\mathrm{O}_{2}$ delivery and utilization during the isokinetic exercise, increased perception of effort, and blood lactate concentration; these observations are all consistent with an increased contribution of the glycolytic energy system to sustain the same absolute intensity. These results suggest a link between exercise intolerance and the decreased ability to produce force.
\end{abstract}

Novelty

- We provided the first characterization of the effects of prolonged stretching on the metabolic response during severe cycling.

- Stretching reduced maximal force and augmented muscle activation, which in turn increased the metabolic response to sustain exercise.

Key words: oxygen consumption, $\dot{\mathrm{VO}}_{2}$ slow component, exercise tolerance, stretching, muscle stretching, loss of efficiency, muscle fatigue, $\dot{V}_{2}$ kinetics, NIRS, EMG.

Résumé : Nous testons l'hypothèse selon laquelle l'étirement statique, qui est une intervention ponctuelle fatigante mais non métabolique, réduit la tolérance à l'exercice en augmentant l'activation musculaire et en influant sur la bioénergétique musculaire au cours d'un exercice de pédalage dans le domaine d'intensité « sévère ». Dix hommes actifs $(24 \pm 2 \mathrm{ans}, 74 \pm 11 \mathrm{~kg}, 176 \pm$ $8 \mathrm{~cm}$ ) participent à des tests de pédalage à intensité constante identique dont deux sont effectués dans des conditions de contrôle et deux après l'étirement, ce qui suscite une réduction de $5 \%$ de la puissance maximale de sprint isocinétique. Nous mesurons : (i) la consommation d'oxygène, (ii) l'électromyogramme, (iii) la désoxyhémoglobine, (iv) la concentration sanguine de lactate, (v) le délai d'épuisement et (vi) la perception de l'effort. Enfin, la cinétique de la consommation d'oxygène et de la désoxyhémoglobine est évaluée. La réduction de la force après l'étirement est accompagnée d'une activation musculaire accrue à une charge de travail donnée ( $p=0,025)$ et d'une réduction significative de délai d'épuisement $(p=0,002)$. Le délai jusqu'au pic de la consommation d'oxygène est réduit par l'étirement $(p=0,034)$, ce qui suggère une influence de l'augmentation de l'activation musculaire sur la cinétique de la consommation d'oxygène. De plus, l'étirement est associé à un décalage entre la livraison et l'utilisation d' $\mathrm{O}_{2}$ pendant l'exercice isocinétique, à un accroissement de la perception de l'effort et de la concentration sanguine de lactate; ces observations sont toutes compatibles avec une contribution accrue du système d'énergie glycolytique pour maintenir la même intensité absolue. Ces résultats suggèrent un lien entre l'intolérance à l'exercice et la diminution de la capacité à produire de la force. [Traduit par la Rédaction]

Les nouveautés

- Nous présentons la première caractérisation des effets d'un étirement prolongé sur la réponse métabolique lors de séances sévères de pédalage.

- L'étirement a réduit la force maximale et augmenté l'activation musculaire, ce qui a augmenté la réponse métabolique pour soutenir l'exercice.

Mots-clés : consommation d'oxygène, composante lente du $\dot{V} \mathrm{O}_{2}$, tolérance à l'exercice, étirement, étirement musculaire, perte d'efficacité, fatigue musculaire, cinétique $\mathrm{VO}_{2}$, NIRS, EMG.

Received 20 December 2019. Accepted 6 March 2020.

A.L. Colosio, M. Teso, and S. Pogliaghi. Department of Neurosciences, Biomedicine and Movement Sciences, University of Verona, Via Casorati 43, Verona 37131, Italy.

Corresponding author: Silvia Pogliaghi (email: silvia.pogliaghi@univr.it).

Copyright remains with the author(s) or their institution(s). Permission for reuse (free in most cases) can be obtained from copyright.com. 


\section{Introduction}

During whole-body exercise at constant-load in the moderate domain, oxygen consumption $\left(\mathrm{VO}_{2}\right)$ adapts to the energetic demands of locomotor and ventilatory muscles within $3 \mathrm{~min}$ (Poole and Jones 2012). If relative intensity rises above the gas exchange threshold (GET), approximately after the third minute of exercise $\dot{\mathrm{V}} \mathrm{O}_{2}$ displays a "slow component" $\left(\dot{\mathrm{VO}}_{2 \mathrm{sc}}\right)$ that is typically interpreted as an increased cost of locomotion for a given exercise intensity (Poole and Jones 2012). In particular, when exercise is performed between the metabolic rates associated with the GET and the respiratory compensation point (RCP; i.e., heavy intensity domain) (Keir et al. 2015, 2018), $\dot{\mathrm{V}} \mathrm{O}_{2 \mathrm{sc}}$ tends to a steady-state; however, when effort rises above the RCP (i.e., severe exercise domain) a steady-state is no longer achievable and $\mathrm{VO}_{2}$ increases towards maximum oxygen consumption $\left(\dot{V} \mathrm{O}_{\text {max }}\right)$ (Jones et al. 2011). The magnitude of $\dot{V O}_{2 s c}$ is considered linked with exercise intolerance and fatigue (Grassi et al. 2015). Therefore, during the past 40 years many researchers have focused their attention on clarifying the physiological bases of $\dot{V O}_{2 s c}$ (Jones et al. 2011). Two main theories have been proposed to explain the physiological origin of $\dot{V} \mathrm{O}_{2 \mathrm{sc}}$ : (i) decreased metabolic stability of type I muscle fibres associated with increased $\mathrm{O}_{2}$ cost of adenosine triphosphate (ATP) resynthesis and/or increased ATP cost of contraction (Jones et al. 2011; Grassi et al. 2015); and (ii) recruitment of fast-fatigable intrinsically inefficient type II muscle fibres to obtain/maintain the external power output (PO) above a certain intensity threshold (e.g., RCP) (Jones et al. 2011; Poole and Jones 2012; Grassi et al. 2015). However, the exact physiological mechanisms underpinning $\dot{V O}_{2 s c}$ remain elusive, one of the reasons being the difficulty to selectively affect either metabolic stability or type II fibre recruitment in human models. In fact, the different manipulations used in interventional studies (e.g., speed of movement, intensity modulation, aerobic training, priming exercise, nutritional interventions) affect to some extent both metabolic stability and fibre recruitment (Jones et al. 2011).

An interesting approach to selectively augment fibre recruitment while trying to avoid the perturbation of metabolic stability is acute, nonmetabolic fatigue that reduces the ability of muscles to produce force. Among the interventions able to cause acute nonmetabolic fatigue, a promising model could be static stretching, which can impair force production as result of prolonged nervous stimulation (Trajano et al. 2017). It was broadly documented that stretching, particularly when positions are maintained for more than $60 \mathrm{~s}$, can impair maximal force in many different tasks and conditions for a period lasting up to $1 \mathrm{~h}$ (Behm et al. 2016). Given that no effort is required to perform stretching, and that force impairment after stretching is mostly caused by neural mechanisms (Trajano et al. 2017), this would be a particularly convenient model to acutely reduce force and investigate the link between muscle activity and metabolism. Indeed, recent studies (Esposito et al. 2012) documented that when maximal force was acutely reduced by stretching, the oxygen cost of locomotion increases both during ramp incremental (exercise modality in which the $\dot{V O}_{2 s c}$ is defined as "excess $\dot{V} \mathrm{O}_{2}$ " (Grassi et al. 2015)) and constant-load exercises (Esposito et al. 2012; Limonta et al. 2015). However, the above studies did not specifically investigate the underpinnings of $\dot{V} \mathrm{O}_{2 \mathrm{sc}}$ and were, therefore, lacking measures to investigate the link between muscle activation and increased $\dot{V} \mathrm{O}_{2}$ (e.g., electromyography (EMG)). In a recent study from our group, the effects of stretching on the $\mathrm{VO}_{2}$ response during ramp incremental cycling were described while also implementing measures of muscle excitation (Colosio et al. 2019). We found that when muscle force is acutely impaired by stretching muscle excitation also increases in unison with an increased cost of locomotion (i.e., $\dot{V} \mathrm{O}_{2}$ at a given absolute workload).

The above findings, in an incremental exercise paradigm, support the existence of a sequence of events (i.e., acute fatigue, increased muscle activation, loss of metabolic efficiency) that causes the $\mathrm{VO}_{2 \mathrm{sc}}$ with increasing exercise intensity. In this context, constant-load exercise represents the ideal model to determine the possible role of increased muscular activation over time (necessary to maintain the same workload when fatigued) in the genesis of the $\dot{V O}_{2 \mathrm{sc}}$. In fact, only under prolonged, constant-load conditions, the increased cost of locomotion (i.e., $\dot{V} \mathrm{O}_{2 \mathrm{sc}}$ ) at a given intensity has the time to fully manifest itself. The confirmation of a connection between fatigue/increased muscle activation and the loss of metabolic efficiency over time during a constant-load exercise paradigm would further support the existence of a causative link.

Accordingly, this study investigated the effects of acute, nonmetabolic fatigue induced by stretching on central and peripheral physiological measures $\left(\dot{V}_{2}\right.$, blood lactate concentration [LA ${ }^{-}$, electromyography (EMG), Near-Infrared Spectroscopy (NIRS)) during constant-load cycling in the severe exercise domain. We hypothesized that stretching $(i)$ would reduce maximal muscle force; (ii) in turn, force loss would translate in increased muscle excitation at a given absolute workload and (iii) increased muscle excitation would reduce exercise tolerance and increase the $\dot{V O}_{2}$ cost of locomotion. Finally, this study will provide the first comprehensive investigation on the effects of static stretching on highintensity constant-load cycling.

\section{Materials and methods}

\section{Participants}

Ten active men gave written informed consent to participate in the study (age: $24 \pm 2$ years, body mass: $74 \pm 11 \mathrm{~kg}$, stature: $176 \pm$ $8 \mathrm{~cm}$ ). Inclusion criteria were male sex and age between 20 and 35 years; exclusion criteria were smoking and any condition that could influence the physiological responses during testing. The study was approved by Departmental Ethics Committee and adhered to the principles of the Declaration of Helsinki. All participants were instructed to avoid physical activity for at least $24 \mathrm{~h}$ before each testing session and followed a standard and individualized food intake prescription before all the testing sessions to minimize variability of glycogen stores and glucose oxidation (i.e., $2 \mathrm{~g}$ of low glycemic index carbohydrates per $\mathrm{kg}$ of body weight, $2 \mathrm{~h}$ before testing; $0.5 \mathrm{~L}$ of water in the $90 \mathrm{~min}$ before testing; restriction from caffeine during the $8 \mathrm{~h}$ before testing).

\section{Experimental protocol}

After medical clearance, participants visited the laboratory on 8 occasions within a maximum of 3 weeks.

On the first 2 visits, subjects familiarized with a test consisting of isokinetic sprints for the determination of the maximal cycling PO. On the third appointment, isokinetic sprints were performed before and after either the control condition (i.e., $40 \mathrm{~min}$ of seated rest, control) or $40 \mathrm{~min}$ of stretching to determine the effect of stretching on the maximal cycling PO. On the fourth visit, subjects performed a ramp incremental test to exhaustion for the determination of the GET, the RCP, and the $\dot{V O}_{2 \max }$. Then, during the last 4 visits participants repeated 4 identical constant-load trials in the severe exercise intensity domain (at a PO corresponding to $\Delta 60 \%$ between GET and $\dot{V} O_{2 \max }$ ). Randomly, 2 of the constant-load trials were carried out in control conditions and 2 of the constantload trials were done after $40 \mathrm{~min}$ of stretching. A schematic representation of the protocol is provided in Fig. 1.

All of the tests were conducted at the same time of the day in an environmentally controlled laboratory $\left(22-25{ }^{\circ} \mathrm{C}, 55 \%-65 \%\right.$ relative humidity) on an electromagnetically braked cycle ergometer (Sport Excalibur; Lode, Groningen, Netherlands). Ergometer position was chosen during the first familiarization visit and recorded for the successive appointments. 
Fig. 1. Schematic representation of the overall protocol (above), and of the single testing sessions (below). GET, gas exchange threshold; PO, power output; $\dot{V}_{2}$, oxygen consumption; $\dot{V}_{2 \max }$, maximal oxygen consumption.

\section{Overall Protocol}

Sessions 5-6-7-8, randomized order

Control: constant load trial, $10 \mathrm{~min}$

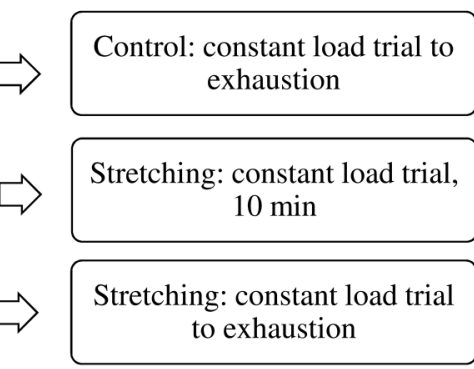

Isokinetic Sprints

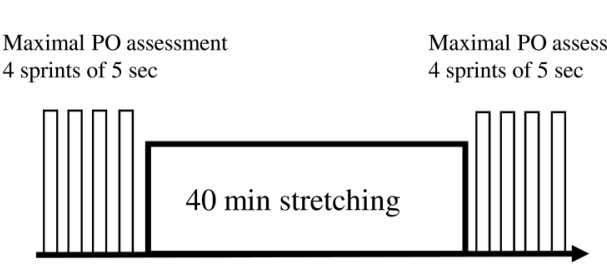

\section{Stretching procedure}

Control consisted in 40 min of resting in a sitting position under the examiner's surveillance.

Six cycles of stretching were used to maximize acute force reduction (Behm et al. 2016). The standardized stretching cycle sequentially involved (i) the quadriceps of the right leg, (ii) the right hamstrings, (iii) the left quadriceps, and (iv) the left hamstrings. Each position was maintained for $80 \mathrm{~s}$ with no recovery between positions. Subjects were continuously encouraged to stretch muscles to the point of discomfort. The total duration of the stretching intervention was about $40 \mathrm{~min}$. Stretching effectiveness in increasing flexibility was measured before and after stretching and control using a sit-and-reach test (Limonta et al. 2015).

\section{Isokinetic maximal sprints}

To assess force reduction after stretching, isokinetic maximal sprints were performed on an electromagnetically braked cycleergometer in isokinetic mode equipped with a pedal force sensor (Sport Excalibur PFM; Lode) as previously described (Colosio et al. 2019). In brief, 2 pedalling frequencies (60 and $120 \mathrm{rpm}$ ) were used to measure velocity-specific peak power as proposed by Cannon et al. (2011). Each sprint session comprised four 5-s maximal sprints, alternating between $60-120-60-120 \mathrm{rpm}$. The 4 maximal sprints were separated by a 2-min passive rest to maximise recovery while limiting the total duration of the sprint session.

\section{Ramp incremental test}

The ramp incremental test consisted of 4-min of baseline cycling at $20 \mathrm{~W}$, followed by increases in PO ranging from 17.5 to $25 \mathrm{~W} / \mathrm{min}$ according to individuals' predicted fitness level with the aim of obtaining a time to exhaustion (TTE) of $\sim 8-12 \mathrm{~min}$ (American College of Sports Medicine 2017) using a method extensively described elsewhere (Pogliaghi et al. 2014). Participants were asked to pick a self-selected cadence in the range of 70$90 \mathrm{rpm}$ and to maintain it throughout all tests. Failure to maintain the indicated cadence within $5 \mathrm{rpm}$ (for longer than $5 \mathrm{~s}$ ) during testing despite strong verbal encouragement was considered as the criterion for exhaustion. Breath-by-breath pulmonary gas ex- change, ventilation, and heart rate were continuously measured using a metabolic cart (Quark $\mathrm{B}^{2}$; Cosmed, Italy) as previously described (De Roia et al. 2012).

\section{Constant-load trials}

After the preliminary ramp incremental test, subjects completed 4 constant-load trials at the PO corresponding to the $60 \% \Delta$ between GET and $\dot{V} \mathrm{O}_{2 \max }$. Two of the constant-load trials were performed after stretching and 2 of the constant-load trials were carried out after control in a randomized order. In each condition, 1 constant-load trial lasted $10 \mathrm{~min}$ while the other was performed to exhaustion to allow recording of TTE after control and stretching. Constant-load trials were preceded by a $4-\mathrm{min}$ warm-up at $20 \mathrm{~W}$ in which cycling cadence was limited to $30 \mathrm{rpm}$ to minimize any metabolic activation that could influence the effects of stretching and the physiological response at the onset of exercise. Throughout the test, subjects kept the same constant rpm selected during the ramp incremental test and the same bike position selected during the sprint test.

Surface EMG of the right vastus lateralis and biceps femoris muscles were continuously recorded by means of a wireless system (Wave wireless EMG; Cometa, Milan, Italy). A pair of surface $\mathrm{Ag} / \mathrm{AgCl}$ electrodes (Blue sensor, Ambu, Ballerup, Denmark) was attached to the skin with a 2-cm inter-electrode distance. The electrodes were placed longitudinally with respect to the underlying muscle fibres arrangement, according to the recommendations by Surface EMG for Non-Invasive Assessment of Muscles (Hermens et al. 2000). Before electrode application, the skin was shaved, scratched with sandpaper, and cleaned with alcohol to minimize impedance. Semi-permanent ink marks allowed consistent re-positioning of the electrodes between sessions. The EMG transmitter connected to the electrodes was well secured with adhesive tape to avoid movement-induced artifacts.

$\dot{V} \mathrm{O}_{2}$, ventilation $(\dot{V E})$, respiratory exchange ratio, and heart rate data were measured with the same method described for the ramp incremental test. During each constant-load trial, capillary blood samples $(20 \mu \mathrm{L})$ were drawn from the earlobe in the last $30 \mathrm{~s}$ 
of warm-up, during the first, third, fifth, seventh, and tenth minutes, and then every $5 \mathrm{~min}$ of the trial to exhaustion. Moreover, blood samples were drawn at the first, third, fifth, and seventh minutes after exhaustion. Samples were immediately analyzed to measure [La-] (Biosen C-Line; EKF Diagnostics, Barleben, Germany).

Deoxygenation of the left vastus lateralis was evaluated in microcirculation using a quantitative NIRS (Oxiplex TSTM; ISS, Champaign, Ill., USA) that provided continuous measurement (sampling frequency $1 \mathrm{~Hz}$ ) of the absolute concentrations $(\mu \mathrm{mol} / \mathrm{L})$ of deoxyhemoglobin concentration ([HHb]). After shaving, cleaning, and drying of the skin area, the NIRS probe was positioned longitudinally on the belly of the vastus lateralis muscle at $\sim 15 \mathrm{~cm}$ above the patella, attached to the skin with a bi-adhesive tape, and secured with elastic bandages around the thigh. The device was calibrated before each test after a warm-up of at least $30 \mathrm{~min}$ as per manufacturer recommendations.

Finally, perceptual responses to exercise was monitored using a 0-100 rating of perceived exertion (RPE) scale (Borg and Kaijser 2006). The scale was displayed to the participants during baseline, every 5 min during the constant-load trials, and immediately after exhaustion.

\section{Data analysis}

\section{Isokinetic sprints test}

Crank torque was measured independently from the 2 crank arms by strain gauge transducers (maximal recordable force $2000 \mathrm{~N},<0.5 \mathrm{~N}$ resolution, and measurement uncertainty of $<3 \%$ ). Angular velocity of the crank was recorded every $2^{\circ}$ using 3 independent sensors sampling in series with uncertainty of measurement $\leq 1 \%$. Overall power for each pedalling cycle was calculated as the sum of the left and the right crank as resulted by the pedal force measurement analysis software. The initial and the last pedalling cycles of each sprint were excluded from computation. Then, maximal power expressed during each pedalling cycle was detected and cycles were averaged to obtain a mean peak PO for every sprint. Finally, mean peak PO of the 2 repetitions of the 60 and $120 \mathrm{rpm}$ sprints performed at either pre- or post-intervention were averaged and the relative percentage of change between pre- and post-conditions were calculated.

\section{Ramp incremental test}

For the gas exchange variables, aberrant data-points that lay 3 SD from the local mean were removed, and trials were linearly interpolated on a 1-s basis and then averaged every $5 \mathrm{~s} . \dot{V} \mathrm{O}_{2 \max }$ was determined as the highest $\dot{V} \mathrm{O}_{2}$ obtained over a 10-s interval (Fontana et al. 2015). GET and RCP were determined with the standard technique from gas exchange variables by 3 blinded expert reviewers as detailed elsewhere (Fontana et al. 2015). Briefly, GET was determined by visual inspection as the $\mathrm{VO}_{2}$ at which $\mathrm{CO}_{2}$ output began to increase out of proportion in relation to $\dot{V O}_{2}$, with a systematic rise in the $\dot{V} \mathrm{E}-\mathrm{to}-\dot{\mathrm{V}} \mathrm{O}_{2}$ relation and end-tidal $\mathrm{PO}_{2}$ whereas the ventilatory equivalent of carbon dioxide production $\left(\dot{V} \mathrm{CO}_{2}\right)\left(\dot{\mathrm{V}} / \mathrm{VCO}_{2}\right)$ and end-tidal $\mathrm{PCO}_{2}$ is stable (Beaver et al. 1986). RCP was determined as the point where end-tidal $\mathrm{PCO}_{2}$ began to fall after a period of isocapnic buffering (Whipp et al. 1989). This point was confirmed by examining $\dot{\mathrm{V}} / \mathrm{VCO}_{2}$ plotted against $\dot{\mathrm{V}} \mathrm{O}_{2}$ and by identifying the second breakpoint in the $\dot{V} \mathrm{E}-\mathrm{to}-\dot{V} \mathrm{O}_{2}$ relation. $\dot{V O}_{2 \max }$ was determined as the highest $\dot{V} \mathrm{O}_{2}$ obtained over a 10 -s interval. Finally, we determined the constant workload equivalent to the specific severe $\left(60 \% \Delta\right.$ between GET and $\left.\dot{V O}_{2 \max }\right) \dot{V O}_{2}$ target. To this aim, the $\mathrm{VO}_{2} / \mathrm{W}$ relationship identified with the incremental test was left-shifted to account for the individual mean response time. Briefly, the mean response time was determined as the time interval between the onset of the incremental portion of the exercise (time $=0$ ) and the increase of the $\mathrm{VO}_{2}$ signal above baseline. It was determined as the $x$ coordinate of the intersection of the forward extrapolation of the baseline $\dot{V O}_{2}$ and the backwards extrapolation of the linear $\dot{V} \mathrm{O}_{2}$-time relationship below the GET (Fontana et al. 2015).

\section{Constant-load trials}

The raw EMG signal was rectified and smoothed using a fourthorder band-pass Butterworth digital filter with a frequency range set between 20 and $500 \mathrm{~Hz}$. Root mean square (RMS) was calculated every second and averaged at 30-s intervals from the raw signal and was used as an index of the total muscle excitation for vastus lateralis $\left(\mathrm{RMS}_{\mathrm{VL}}\right)$ and biceps femoris $\left(\mathrm{RMS}_{\mathrm{BF}}\right)$ (Vigotsky et al. 2018). Thereafter, the RMS recorded during the last $2 \mathrm{~min}$ of $20 \mathrm{~W}$ baseline for each test was used to normalize the constant-load trials and expressed as multiples of baseline.

TTE was calculated as the total duration of exercise from workload onset to failure.

$\dot{V} \mathrm{O}_{2}$ during constant-load trials was cleaned and interpolated using the same procedure described for the ramp incremental test. Then, data of the 2 constant-load trials performed in each condition were mediated to reduce breath-by-breath signals variability. Finally, 30-s means were calculated.

Net $\left[\mathrm{La}^{-}\right]$accumulation during constant-load trials was calculated as the difference between $\left[\mathrm{La}^{-}\right]$at a specific timepoint and the $\left[\mathrm{La}^{-}\right]$during cycling at $20 \mathrm{~W}$. The highest value after exercise end was considered as the peak of $\left[\mathrm{La}^{-}\right]$.

NIRS-derived $[\mathrm{HHb}]$ response during constant-load trials was time aligned with the onset of exercise transition, treated by subtracting the steady-state value measured during the last $2 \mathrm{~min}$ of warm-up, and then averaged at 30-s bins.

\section{$\dot{\mathrm{V}} \mathrm{O}_{2},[\mathrm{HHb}]$ kinetics, and $\dot{\mathrm{V}} \mathrm{O}_{2 \mathrm{sc}}$}

Using 1-s bins data, the on-transient responses to exercise of $\dot{V O}_{2}$ was modelled as follows: first, the $\mathrm{VO}_{2}$ response from -60 up to $180 \mathrm{~s}$ (time 0 being exercise onset) was preliminarily characterized with a 2-component model (linear + exponential), integrated by a Heaviside function, after the exclusion of the data points of the initial $20 \mathrm{~s}$ of exercise that corresponded to the cardiodynamic phase (Murias et al. 2011a). With this approach, we derived the initial parameters for the primary component. Then, the complete on-transient responses to exercise of $\dot{V O}_{2}$ were modelled from the onset of workload to the end of the tenth minute (or to exhaustion for tests that lasted less than $10 \mathrm{~min}$ after stretching) using the following 2-component exponential equation integrated by a Heaviside function (De Roia et al. 2012):

$$
Y_{(t)}=Y_{\mathrm{bsln}}+\operatorname{AMP}_{\mathrm{p}}\left[1-e^{-\left(t-\mathrm{TD}_{\mathrm{p}}\right) / \tau_{\mathrm{p}}}\right]+\mathrm{AMP}_{\mathrm{sc}}\left[1-e^{-\left(t-\mathrm{TD}_{\mathrm{sc}}\right) / \tau_{\mathrm{sc}}}\right]
$$

where $Y_{(t)}$ represents the increase in $\dot{V} \mathrm{O}_{2}$ at the onset of exercise; $\mathrm{Y}_{\text {blsn }}$ is the baseline $\mathrm{VO}_{2}$ value recorder during the $4 \mathrm{~min}$ of $20 \mathrm{~W}$ cycling; $\mathrm{AMP}_{\mathrm{p}}$ and $\mathrm{AMP}_{\mathrm{sc}}$ represent the amplitude of the $\mathrm{VO}_{2}$ response above the baseline value of the primary and the slow component, respectively; and $\tau_{\mathrm{p}}, \tau_{\mathrm{sc}}, \mathrm{TD}_{\mathrm{p}}$, and $\mathrm{TD}_{\mathrm{sc}}$ are the time constant and the time delay of the response for each component, respectively. The mean response time (MRT) was then calculated as the sum of $\tau+$ TD. Furthermore, we calculated the time requested to reach $\dot{V} \mathrm{O}_{2 \max }$ during constant-load trials by resolving on the individual fitting of $\mathrm{VO}_{2}$ data for the time coordinate corresponding to $\dot{\mathrm{VO}}_{2 \max }$.

[HHb] signal was fitted on a time window of -60 to $180 \mathrm{~s}$ (time 0 being exercise onset) using a 2-component model (linear + exponential), integrated by a Heaviside function, as previously described (De Roia et al. 2012).

Finally, $[\mathrm{HHb}]$ and $\dot{V}_{2}$ data were normalized with $0 \%$ corresponding to the value recorded while cycling at $20 \mathrm{~W}$ baseline and $100 \%$ reflecting the maximal response in the 180 -s window and expressed as $\Delta[\mathrm{HHb}]$ and $\Delta \dot{V} \mathrm{O}_{2}$. Individualized 1-s $\Delta[\mathrm{HHb}]$ and $\Delta \dot{V} \mathrm{O}_{2}$ were time-aligned by left-shifting the $\dot{V}_{2}$ data by 20 s (i.e., the typical duration of the cardiodynamic phase in young individ- 
uals (Murias et al. 2011b). Then, the ratio between $\Delta[\mathrm{HHb}] / \Delta \dot{V}_{2}$ was calculated during the first $180 \mathrm{~s}$ of exercise to express the fractional muscle $\mathrm{O}_{2}$ extraction required to sustain a given net increment of $\dot{V O}_{2}$ (De Roia et al. 2012). Finally, the following indexes were calculated: $\Delta[\mathrm{HHb}] / \Delta \dot{V} \mathrm{O}_{2}$ area under the curve (AUC), as the integral of the total mismatch between $\mathrm{O}_{2}$ delivery and utilization (i.e., index values > 1); $\Delta[\mathrm{HHb}] / \Delta \dot{V} \mathrm{O}_{2}$ peak, as the maximal value reached within the $180 \mathrm{~s} ; \Delta[\mathrm{HHb}] / \Delta \dot{V} \mathrm{O}_{2}$ time to peak, as the time requested to reach the peak in $\Delta[\mathrm{HHb}] / \Delta \dot{V} \mathrm{O}_{2}$. Moreover, given that these time-resolved values are typically implemented during steady-state condition, an overall quantification of the increase in fractional muscle $\mathrm{O}_{2}$ extraction required to sustain a given net increment in $\dot{V O}_{2}$ during the primary phase of exercise was calculated by dividing the amplitudes of the response in $\dot{V O}_{2}$ and $[\mathrm{HHb}]$ between the onset of exercise and the onset of the slow component: overall $\Delta[\mathrm{HHb}] / \Delta \dot{V} \mathrm{O}_{2}$ (Tam et al. 2018).

\section{Statistics}

After assumptions verification (i.e., normality, homogeneity of variance), 2-way repeated-measures ANOVA was applied to compare flexibility values (pre- and post-sit-and-reach after control/ stretching). Pre- and post-peak POs measured during isokinetic sprints at 60 and 120 rpm were compared pre- and post-stretching, using a 2-way repeated-measures ANOVA (time $\times$ pedalling frequency).

For constant-load trials, 2-way repeated-measures ANOVAs were performed to compare $\dot{V}_{2}$, net [La $\left.{ }^{-}\right], \mathrm{RPE},[\mathrm{HHB}], \mathrm{RMS}_{\mathrm{VL}}$, and $\mathrm{RMS}_{\mathrm{BF}}$ between conditions over time (time $\times$ condition). Post hoc analyses were performed using the Holm-Sidak test. Student's $t$ test was applied to compare between conditions the TTE, parameters of $\dot{V} \mathrm{O}_{2}$ and [HHb] kinetics ( $\tau$, TD, and MRT), time to $\dot{V} \mathrm{O}_{2 \text { max }}$, $\Delta[\mathrm{HHb}] / \Delta \dot{V} \mathrm{O}_{2}$ AUC, $\Delta[\mathrm{HHb}] / \Delta \dot{V} \mathrm{O}_{2}$ peak, $\Delta[\mathrm{HHb}] / \Delta \dot{V} \mathrm{O}_{2}$ time to peak, and overall $\Delta[\mathrm{HHb}] / \Delta \dot{V} \mathrm{O}_{2}$.

Data are presented as means \pm SD. $\alpha$ was set in advance at the 0.05 level and significance was accepted when $p<\alpha$. The $95 \%$ confidence intervals of the $\mathrm{TD}_{\mathrm{p}}$ and $\mathrm{TD}_{\mathrm{sc}}, \tau_{\mathrm{p}}$, and $\tau_{\mathrm{sc}}$ of $\dot{V O}_{2}$ kinetics, and of TD and $\tau$ of $[\mathrm{HHb}]$ kinetics were calculated based on the asymptotic intervals of the nonlinear parameters resulting from the fitting (Field et al. 2012). Effect sizes of the differences between control and stretching were also reported (Cohen's $d$, ranked as trivial (0-0.19), small (0.20-0.49), medium (0.50-0.79), and large $(\geq 0.80))$ as objective and standardized measures to quantifying the magnitude of difference after stretching versus control (Cumming 2014). In Cohen's effect size calculation, the SD in the control condition was used to standardize the mean difference for each contrast (Field et al. 2012). Moreover, generalized eta squared $\left(\eta_{G}{ }^{2}\right)$ were calculated to quantify the effects sizes of different independent variables during the constant-load trials (Olejnik and Algina 2003; Bakeman 2005). Based on an expected SD of breath-bybreath $\dot{V}_{2}$ measurements for steady-state exercise equal to $2.5 \%$, and a minimum detectable change in $\dot{V O}_{2}$ of $100-170 \mathrm{~mL} / \mathrm{min}$ at a $\dot{V} \mathrm{O}_{2}$ of 2.1 to $3.5 \mathrm{~L} / \mathrm{min}$ (Keir et al. 2015), the minimum sample size to obtain a power of 0.8 was 6 individuals. All statistical analyses were performed using SigmaPlot, version 12 (Systat Software Inc, USA).

\section{Results}

Flexibility, as measured by sit-and-reach test, was not significantly different at baseline between stretching and control and significantly improved only after stretching (pre-stretching: $+0.3 \pm$ $6.5 \mathrm{~cm}$ vs post-stretching: $+6.1 \pm 5.9 \mathrm{~cm}, p<0.001, d=+0.89$; pre-control: $+0.7 \pm 5.1 \mathrm{~cm}$ vs post-control: $+0.8 \pm 4.9 \mathrm{~cm}, p=0.784$, $d=+0.02$ ). The peak PO measured during isokinetic sprints prestretching was reduced after the intervention by $\approx 5 \%$ (Table 1 ). ANOVA revealed a significant main effect of time $(p \leq 0.001)$ and pedalling frequency $(p \leq 0.001)$, with no interaction $(p=0.885)$. Post hoc analysis confirmed that peak PO was significantly re-
Table 1. Mean \pm SD peak power output during isokinetic sprints at pre- and post-static stretching.

\begin{tabular}{llllll}
\hline $\begin{array}{l}\text { Pedalling } \\
\text { frequency } \\
(\mathrm{rpm})\end{array}$ & $\begin{array}{l}\text { Pre-stretching } \\
(\mathrm{W})\end{array}$ & $\begin{array}{l}\text { Post-stretching } \\
(\mathrm{W})\end{array}$ & $\Delta \%$ & $p$ & $d$ \\
\hline 60 & $670 \pm 132$ & $626 \pm 107^{*}$ & $-6.0 \pm 5.3$ & 0.009 & -0.33 \\
120 & $903 \pm 86$ & $863 \pm 112^{*}$ & $-4.6 \pm 4.8$ & 0.014 & -0.46 \\
\hline
\end{tabular}

*Significant differences between pre- and post-stretching values.

duced by stretching both during the $60 \mathrm{rpm}$ and the $120 \mathrm{rpm}$ sprints (Table 1 ).

The mean $\dot{V} \mathrm{O}_{2 \max }$ and peak $\mathrm{PO}$ of subjects measured at the end of the ramp incremental test were $3505 \pm 375 \mathrm{~mL} / \mathrm{min}$ and $315 \pm$ $26 \mathrm{~W}$, respectively. GET and RCP were detected at a $\dot{V O}_{2}$ of $2155 \pm$ $355 \mathrm{~mL} / \mathrm{min}$ and $2900 \pm 472 \mathrm{~mL} / \mathrm{min}$. The calculated target $\mathrm{VO}_{2}$ and PO for the $\Delta 60 \%$ constant-load trials were $3030 \pm 411 \mathrm{~mL} / \mathrm{min}$ and $232 \pm 29 \mathrm{~W}(74 \% \pm 7 \%$ of the peak PO), respectively. As expected under the nonsteady-state conditions of the severe-intensity domain, the contribution of the $\dot{V}_{2}$ slow component raised the actual experimental $\dot{V O}_{2}$ above the initially predicted target so that values close to $\dot{V} \mathrm{O}_{2 \max }$ were measured in the last $20 \mathrm{~s}$ of the 10-min trials. In 1 subject only, the target intensity turned out to fall clearly below the desired severe domain (i.e., both $\dot{V O}_{2}$ and $\left[\mathrm{La}^{-}\right]$were stable over time after the tenth minute of exercise and TTE exceeded $40 \mathrm{~min}$ ). Therefore, for this subject the constantload trials performed until that moment were repeated at $+20 \mathrm{~W}$ after a wash-out period of 3 weeks to assure a metabolic intensity corresponding to the desired severe-intensity domain.

The TTE for the constant-load trials of the control condition was $839 \pm 200 \mathrm{~s}(14: 19 \pm 3: 20 \mathrm{~min}: \mathrm{s})$. Stretching significantly affected this parameter, which was reduced to $743 \pm 166 \mathrm{~s}$ (12:23 \pm 2:46 min:s, $p=0.002, d=-0.48)$. Reduced TTE was associated with increased levels of $\mathrm{RMS}_{\mathrm{VL}}$, [HHb], peak net [LA'], and perceived exertion at exhaustion (Fig. 2). In fact, $\mathrm{RMS}_{\mathrm{VL}}$, [HHb], peak net [ $\left.\mathrm{La}^{-}\right]$, and perceived exertion were significantly higher in the stretching versus the control condition (main effect of time $-\mathrm{RMS}_{\mathrm{VL}}: p<0.00, \eta_{\mathrm{G}}{ }^{2}$ : $0.30,[\mathrm{HHb}]: p<0.00, \eta_{\mathrm{G}}{ }^{2}: 0.65$, peak net $\left[\mathrm{La}^{-}\right]: p<0.00, \eta_{\mathrm{G}}{ }^{2}: 0.99$, perceived exertion: $p<0.00, \eta_{\mathrm{G}}^{2}: 0.990$; main effect of condition $\mathrm{RMS}_{\mathrm{VL}}: p=0.025, \eta_{\mathrm{G}}^{2}: 0.44$, [HHb]: $p=0.011, \eta_{\mathrm{G}}{ }^{2}: 0.53$, net [LA ${ }^{-}$: $p=0.023, \eta_{\mathrm{G}}{ }^{2}: 0.49$, perceived exertion: $p=0.003, \eta_{\mathrm{G}}{ }^{2}: 0.50$; time $\times$ condition interactions - $\mathrm{RMS}_{\mathrm{VL}}: p<0.001, \eta_{\mathrm{G}}^{2}: 0.70,[\mathrm{HHb}]$ : $p=0.007, \eta_{\mathrm{G}}^{2}: 0.34$, net $\left[\mathrm{LA}^{-}\right]: p=0.221, \eta_{\mathrm{G}}{ }^{2}: 0.01$, perceived exertion: $\left.p=0.044, \eta_{\mathrm{G}}{ }^{2}: 0.01\right)$. On the contrary, there were no changes in $\dot{V} \mathrm{O}_{2}$ and $\mathrm{RMS}_{\mathrm{BF}}$ in stretching versus control (main effect of time - $\mathrm{VO}_{2}: p<0.001, \eta_{\mathrm{G}}{ }^{2}: 0.99, \mathrm{RMS}_{\mathrm{BF}}: p<0.001, \eta_{\mathrm{G}}{ }^{2}: 0.77$; no main effect of condition $-\dot{V} \mathrm{O}_{2}: p=0.864, \eta_{\mathrm{G}}{ }^{2}: 0.01, \mathrm{RMS}_{\mathrm{BF}}: p=0.362, \eta_{\mathrm{G}}{ }^{2}$ : 0.09). $\dot{V} \mathrm{O}_{2}$ kinetics analysis revealed that the time required to reach the $\dot{V O}_{2 \max }$ during constant-load trials was reduced by stretching (control $=709 \pm 183$ vs stretching $639 \pm 197, p=0.034, d=$ $-0.38)$.

Other parameters regarding $\dot{V O}_{2}$ and [HHb] kinetics (e.g., $\tau$, TD, etc.) are presented in Table 2 and Fig. 3 displays the mean signals during the transient phase after control and stretching. $\mathrm{VO}_{2}$ showed no changes between control and stretching in time delay ( $p=0.874, d=+0.04)$, and in $\tau(p=0.066, d=-0.31$, Table 2). On the contrary, stretching reduced the time delay of $[\mathrm{HHb}](p=0.023, d=$ -0.61 , Table 2 and Fig. 3$)$, but no difference was detected in $\tau$ $(p=0.690, d=+0.19)$. Finally, during the transient phase (first $180 \mathrm{~s}$ of exercise), $\Delta[\mathrm{HHb}] / \Delta \dot{V} \mathrm{O}_{2}$ revealed an increased mismatch in oxygen delivery and utilization at the peripheral level after stretching (Fig. 3), corroborated by a significant difference between conditions in $\Delta[\mathrm{HHb}] / \Delta \dot{V} \mathrm{O}_{2}$ peak $(p=0.038, d=+1.01)$, and a larger, yet not significant of $\Delta[\mathrm{HHb}] / \Delta \dot{V} \mathrm{O}_{2} \mathrm{AUC}(p=0.099, d=+0.59$ Table 2 and Fig. 3). 
Fig. 2. The physiological responses during control (black dots) and stretching (white dots) conditions are presented in $30 \mathrm{~s}$ (means \pm SD). \%bas, RMS values are expressed as precentage of the value recorded during the 20-W warm-up phase; BF, biceps femoris; [HHb], deoxyhemoglobin concentration; NIRS, Near-Infrared Spectroscopy; RMS, root mean square; RPE, rating of perceived exertion; VL, vastus lateralis; $\mathrm{VO}_{2}$, oxygen consumption; $\dot{V O}_{2 \max }$, maximal oxygen consumption *, Statistical difference between control and stretching for a given timepoint.
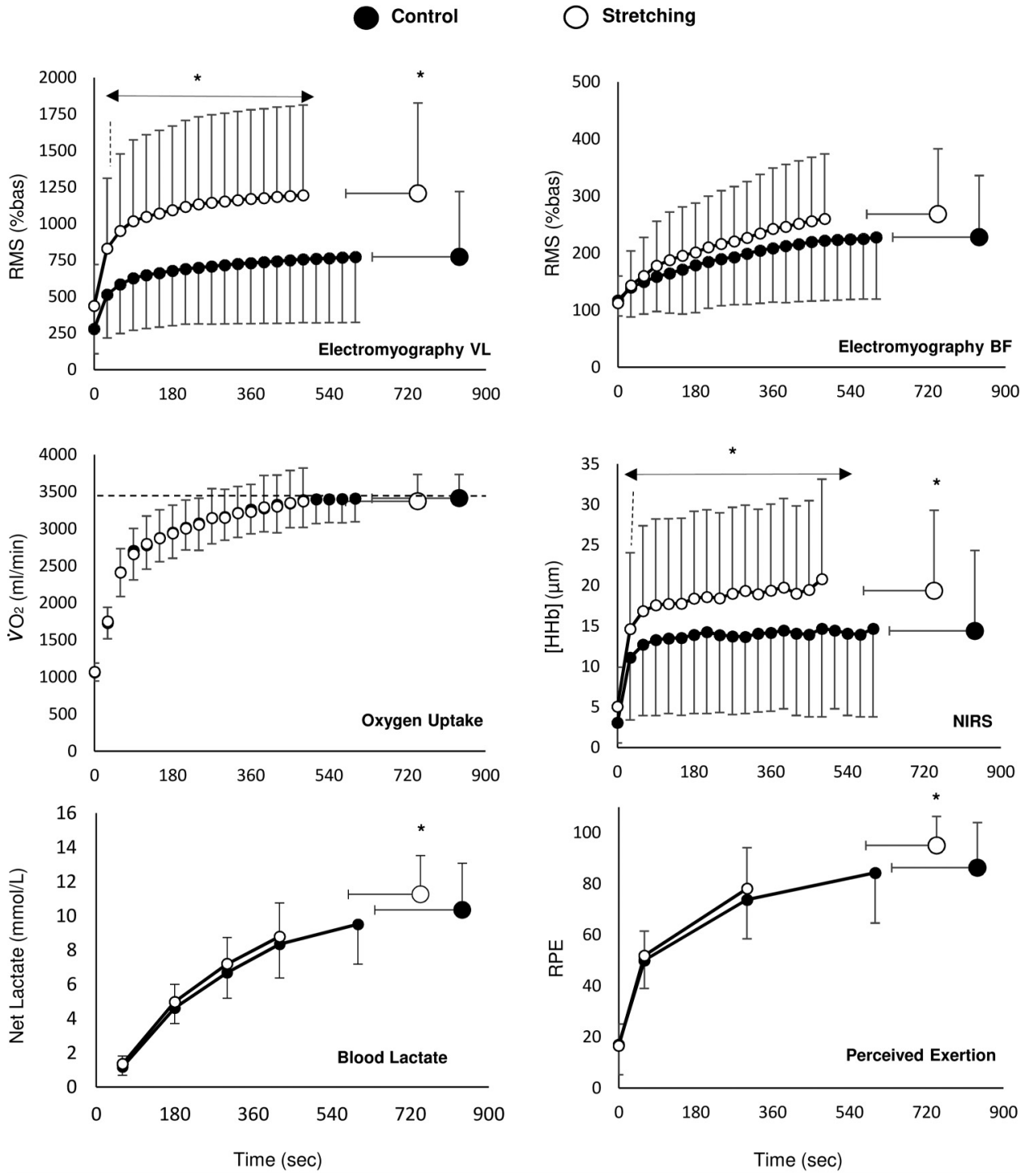

\section{Discussion}

This investigation evaluated the physiological effects of acute nonmetabolic fatigue induced by static stretching on highintensity constant-load cycling performance. We had hypothesized that stretching through a sequence of events, going from a reduction of maximal muscle force, to an increase in muscle excitation at a given absolute workload, to an increase in the $\mathrm{VO}_{2}$ cost of locomotion, would ultimately reduce exercise tolerance. In agreement with our hypothesis, stretching caused significant fatigue, as indicated by the reduction of the maximal PO during isokinetic sprints. In turn, force reduction was accompanied by an augmented muscle excitation at a given workload. Finally, the above sequence of events was associated with a significant reduction in TTE. Contrary to our hypothesis, no changes were detected between control and stretching in the magnitude of $\dot{V} \mathrm{O}_{2 \mathrm{sc}}$ in the time window in which $\dot{V O}_{2 s c}$ is typically investigated (from the $\approx$ second to third to the ninth to tenth minutes). However, the time required to reach $\mathrm{VO}_{2 \max }$ was reduced by stretching, suggesting an influence of the increased muscle excitation on the dynamics of $\mathrm{VO}_{2}$ during constant-load exercise in the severe-intensity do-

main. In support of this, stretching was associated with an increased metabolic instability/mismatch between $\mathrm{O}_{2}$ delivery and $\mathrm{O}_{2}$ utilization during the on-kinetic, an increased perception of effort, and an increased [La-], which are all compatible with an increased contribution of the glycolytic energy system to sustain the same absolute intensity.

The effectiveness of stretching in acutely impairing maximal force/power was previously proven during cycling-specific (Colosio et al. 2019) and nonspecific tasks (Behm et al. 2016). In agreement with the above findings, our study confirmed a reduction of the maximal cycling PO following stretching intervention of a similar order of magnitude (i.e., $\approx 5 \%$ ) (Table 1 ) of that previously described. The physiological causes of the impairment of maximal PO documented after repeated bouts of prolonged stretching remain to be elucidated and supraspinal-, spinal-, or muscle-related mechanisms have all been proposed as possible explanatory causes of reduced maximal force (Trajano et al. 2017). Independently from the cause, a reduction of maximal force may translate in the requirement of higher levels of relative force to maintain the same absolute workload. In turn, this could augment the re- 
Table 2. Kinetic parameters for oxygen consumption and deoxyhemoglobin.

\begin{tabular}{|c|c|c|c|c|}
\hline & Control & Stretching & $p$ & $d$ \\
\hline \multicolumn{5}{|l|}{$\dot{\mathrm{V}} \mathrm{O}_{2}$} \\
\hline Baseline (mL/min) & $787.1 \pm 174.6$ & $790.7 \pm 105.9$ & 0.938 & +0.02 \\
\hline $\operatorname{AMP}_{\mathrm{p}}(\mathrm{mL} / \mathrm{min})$ & $2015.4 \pm 480.1$ & $2045.2 \pm 509.9$ & 0.607 & +0.06 \\
\hline $\mathrm{TD}_{\mathrm{p}}(\mathrm{s})$ & $14.7 \pm 4.8$ & $14.9 \pm 10.7$ & 0.873 & +0.04 \\
\hline $\mathrm{TD}_{\mathrm{p}}, 95 \% \mathrm{CI}(\mathrm{s})$ & $13.2-16.2$ & $13.6-16.1$ & & \\
\hline$\tau_{p}(s)$ & $32.6 \pm 10.5$ & $29.3 \pm 11.2$ & 0.066 & -0.31 \\
\hline$\tau_{\mathrm{p}}, 95 \% \mathrm{CI}(\mathrm{s})$ & $29.8-35.4$ & $26.9-31.8$ & & \\
\hline $\operatorname{MRT}_{\mathrm{p}}(\mathrm{s})$ & $47.3 \pm 10.7$ & $44.2 \pm 12.3$ & 0.116 & -0.29 \\
\hline $\mathrm{AMP}_{\mathrm{sc}}(\mathrm{mL} / \mathrm{min})$ & $752.1 \pm 386.9$ & $756.1 \pm 272.8$ & 0.207 & +0.01 \\
\hline $\mathrm{TD}_{\mathrm{sc}}(\mathrm{s})$ & $152.6 \pm 37.5$ & $155.3 \pm 25.6$ & 0.803 & +0.05 \\
\hline $\mathrm{TD}_{\mathrm{sc}}, 95 \% \mathrm{CI}(\mathrm{s})$ & 146.5-158.7 & 147.1-163.4 & & \\
\hline$\tau_{\mathrm{sc}}(\mathrm{s})$ & $321.9 \pm 149.7$ & $282.9 \pm 111.5$ & 0.333 & -0.26 \\
\hline$\tau_{\mathrm{sc}}, 95 \% \mathrm{CI}(\mathrm{s})$ & $251.9-392.0$ & $147.1-163.4$ & & \\
\hline \multicolumn{5}{|l|}{ [HHb] } \\
\hline Baseline ( $\mu \mathrm{mol}$ & $24.7 \pm 9.4$ & $27.3 \pm 10.3$ & 0.138 & +0.38 \\
\hline $\operatorname{AMP}(\mu \mathrm{mol} / \mathrm{L})$ & $14.8 \pm 9.4$ & $17.3 \pm 12.2$ & 0.359 & +0.26 \\
\hline $\mathrm{TD}(\mathrm{s})$ & $6.9 \pm 3.8$ & $4.6 \pm 3.0$ & 0.023 & -0.61 \\
\hline TD, 95\% CI (s) & $6.2-7.7$ & $4.0-5.3$ & & \\
\hline$\tau(\mathrm{s})$ & $16.8 \pm 6.5$ & $18.0 \pm 4.7$ & 0.689 & +0.19 \\
\hline$\tau, 95 \% \mathrm{CI}(\mathrm{s})$ & $15.7-17.9$ & $16.8-19.2$ & & \\
\hline MRT (s) & $23.7 \pm 9.0$ & $22.6 \pm 7.5$ & 0.767 & -0.12 \\
\hline \multicolumn{5}{|l|}{$\Delta[\mathbf{H H b}] / \Delta \dot{V} \mathbf{O}_{2}$} \\
\hline AUC & 30.4 & $41.1 \pm 16.1$ & 0.099 & +0.75 \\
\hline Peak & $1.9 \pm 0.5$ & $2.4 \pm 0.4$ & 0.038 & +1.01 \\
\hline Time to & $18.9 \pm 5.8$ & $18.4 \pm 8.5$ & 0.891 & -0.09 \\
\hline $\begin{array}{l}\text { Overall } \Delta[\mathrm{HHb}] / \Delta \mathrm{VO}_{2} \text { ratio } \\
\left(\mu \mathrm{mol} \cdot \mathrm{L}^{-1} \cdot \mathrm{mL}^{-1} \cdot \mathrm{min}^{-1}\right)\end{array}$ & $0.011 \pm 0.008$ & $0.014 \pm 0.012$ & 0.275 & +0.28 \\
\hline
\end{tabular}

Note: Values are presented as means \pm SD or $95 \% \mathrm{CI}$, as indicated. $\tau$, time constant of response; AMP, amplitude; AUC, area under the curve; CI, confidence interval; $[\mathrm{HHb}]$, deoxyhemoglobin concentration; MRT, mean response time; TD, time delay $\mathrm{VO}_{2}$, oxygen consumption. Subscripts: p, primary component; sc, slow component.

cruitment of high-order, fast-fatiguing motor units (progressive recruitment theory (Henneman et al. 1965)) or the frequency of activation of motor units, at the same absolute PO. Both mechanisms are likely to cause an increase in the overall electrical activity of the muscle, as evaluated by surface EMG (Vigotsky et al. 2018).

To our knowledge, our study was the first to examine EMG after stretching during cycling. According to our hypothesis, the impairment of maximal PO documented after stretching translated in augmented muscular excitation of the vastus lateralis (Fig. 2); this finding supports the hypothesis that either the recruitment of higher order motor units or an increased activation frequency were necessary to sustain exercise at the same absolute workload compared with the control condition (Fig. 2). The increase in muscle activation (vs control) manifested clearly within the first minute after the onset of exercise, with no further effect over time. This would support the idea that the increased muscle excitation following stretching was a result of the acute loss in maximal PO rather than to progressive fatigue. Interestingly, our intervention affected the muscle excitation of the vastus lateralis to a larger extent than that of the biceps femoris (Fig. 2). We speculate that this difference may be due to a larger effectiveness of stretching on the extensor of the knee compared with the flexor (i.e., mostly for the different anatomical insertion of these muscles). Moreover, a smaller contribution of the biceps femoris than the vastus lateralis during cycling, particularly in a sample of noncyclist subjects, may have influenced these results.

Contrary to our hypothesis, increased muscle excitation did not influence the $\dot{V} \mathrm{O}_{2 \mathrm{sc}}$. Previous studies on the $\dot{V} \mathrm{O}_{2 \mathrm{sc}}$ response following fatigue have led to inconsistent results: the $\dot{V} \mathrm{O}_{2 \mathrm{sc}}$ was augmented (Colosio et al. 2019), unaffected (Hopker et al. 2016), or even diminished (Deley et al. 2006). This may be explained by the heterogeneity of the fatiguing protocols adopted in the different studies (e.g., stretching, dropjumps, electrical stimulation, etc.), by the exercise domain investigated (heavy/steady-state vs severe/ nonsteady-state) and by the time window considered and the analysis strategy (e.g., fitting, integral, etc.). Furthermore, in the nonsteady-state, severe-intensity exercise used in our study, $\dot{V} \mathrm{O}_{2}$ rapidly projects to $\dot{\mathrm{V}} \mathrm{O}_{2 \max }$ (Fig. 2), reaching this upper ceiling within $\sim 10 \mathrm{~min}$. Under these conditions the potential for an increase in the $\dot{V} \mathrm{O}_{2 s c}$ (i.e., the difference in $\dot{V} \mathrm{O}_{2}$ between the $\approx$ second to third and the ninth to tenth minutes) in response to stretching may have been too small to be measurable. In support of this view, the magnitude of the stretching effect measured in our study is consistent with the rather small increase in $\dot{V O}_{2}(\sim 100-150 \mathrm{~mL} /$ min) reported by Esposito et al. (2012) following stretching. Interestingly, however, our data showed a reduction in the time necessary to reach $\dot{V} \mathrm{O}_{2 \max }$ (of $\approx 70 \mathrm{~s}, \approx 10 \%$ ) and augmented peak $\left[\mathrm{La}^{-}\right]$and peak RPE (Fig. 2) following stretching compared with the control condition. These findings appear compatible with a faster projection of $\dot{\mathrm{VO}} \mathrm{O}_{2}$ towards $\dot{\mathrm{V}} \mathrm{O}_{2 \max }$ following stretching, possibly driven by the increased amount of muscle fibres necessary to sustain the same absolute workload, as indicated by the increased EMGof the vastus lateralis in the fatigued condition.

Finally, a mirror intervention of acute fatigue that could provide complementary information on the link between muscle recruitment and $\dot{V} \mathrm{O}_{2 s c}$ is represented by strength training. In this context, Tam et al. trained a group of older people for 2 months with either interval training or resistance training (Tam et al. 2018). A direct comparison with this study is not fully applicable because of the lack of EMG measures and to the fact that anatomical and functional adaptations other than an isolated improvement of muscle strength occurred in Tam's study. Still, the increased muscle strength reported together with a slight decrease of the $\mathrm{VO}_{2 \mathrm{sc}}$ amplitude seem to corroborate a possible role of muscle activation in the genesis of the $\dot{V O}_{2 s c}$ (Tam et al. 2018).

Regarding the metabolic impact of stretching documented with NIRS (Fig. 2), a reduced time delay of the [HHb] kinetics at the onset of exercise (Fig. 3 ) and an augmented peak $[\mathrm{HHb}] / \mathrm{VO}_{2}$ ratio (Fig. 3; Table 2) were observed. These indexes indicate an earlier mismatch between oxygen delivery and oxygen utilization within the working muscles and a larger mismatch during the onkinetics, respectively (Grassi et al. 2003). Both findings are compatible with either an increase in oxygen extraction and/or a lower oxygen delivery in the working muscles. While this is the first study that measured tissues oxygenation levels during cycling after stretching, recent studies showed that stretching can induce temporary ischemia followed by reactive hyperaemia and possibly enhance $\mathrm{O}_{2}$ delivery at the macro- (Venturelli et al. 2019) and micro- (Trajano et al. 2014) circulatory levels. Therefore, while a reduction in muscle perfusion cannot be completely ruled out, it seems unlikely that oxygen availability in the working muscles would be reduced after stretching. Alternatively, stretching may be associated with a faster oxygen extraction caused by a faster and larger disturbance of intracellular homeostasis. The higher deoxygenation during the fatigued condition may be explained by the greater proportion of glycolytic muscle fibres being involved into exercise. In fact, the glycolytic fibres seem to display higher levels of $\mathrm{O}_{2}$ extraction compared with the aerobic fibres at the onset of exercise (Ferreira et al. 2006; Koga et al. 2014), possibly because of a local Bohr effect (Jensen 2004). Interestingly, these speculations appear consistent with the finding of a faster projection of $\dot{V} \mathrm{O}_{2}$ towards $\dot{V} \mathrm{O}_{2 \max }$ following stretching.

In conclusion, prolonged stretching caused acute fatigue as indicated by a reduced maximal PO in isokinetic sprints. Stretchinginduced fatigue, which in turn caused augmented levels of muscle excitation at a given workload when cycling in the severe exercise domain and resulted in a significant reduction in TTE. No changes were detected between control and stretching in the magnitude of $\dot{V} \mathrm{O}_{2 \mathrm{sc}}$ per se; however, the time required to reach $\dot{\mathrm{VO}}_{2 \max }$ was 
Fig. 3. The oxygen consumption $\left(\dot{V}_{2}\right)$; top panel), deoxyhemoglobin concentration ([HHb]; middle panel), and $\Delta[\mathrm{HHb}] / \Delta \dot{V} \mathrm{O}_{2}($ bottom panel) kinetics during the first $180 \mathrm{~s}$ after exercise onset are presented during control (black dots) and stretching (white dots). $\tau$, time constant of response; AUC, area under the curve; TD, time delay; MRT, mean response time. *, Statistical difference between control and stretching for a given value of the kinetics.
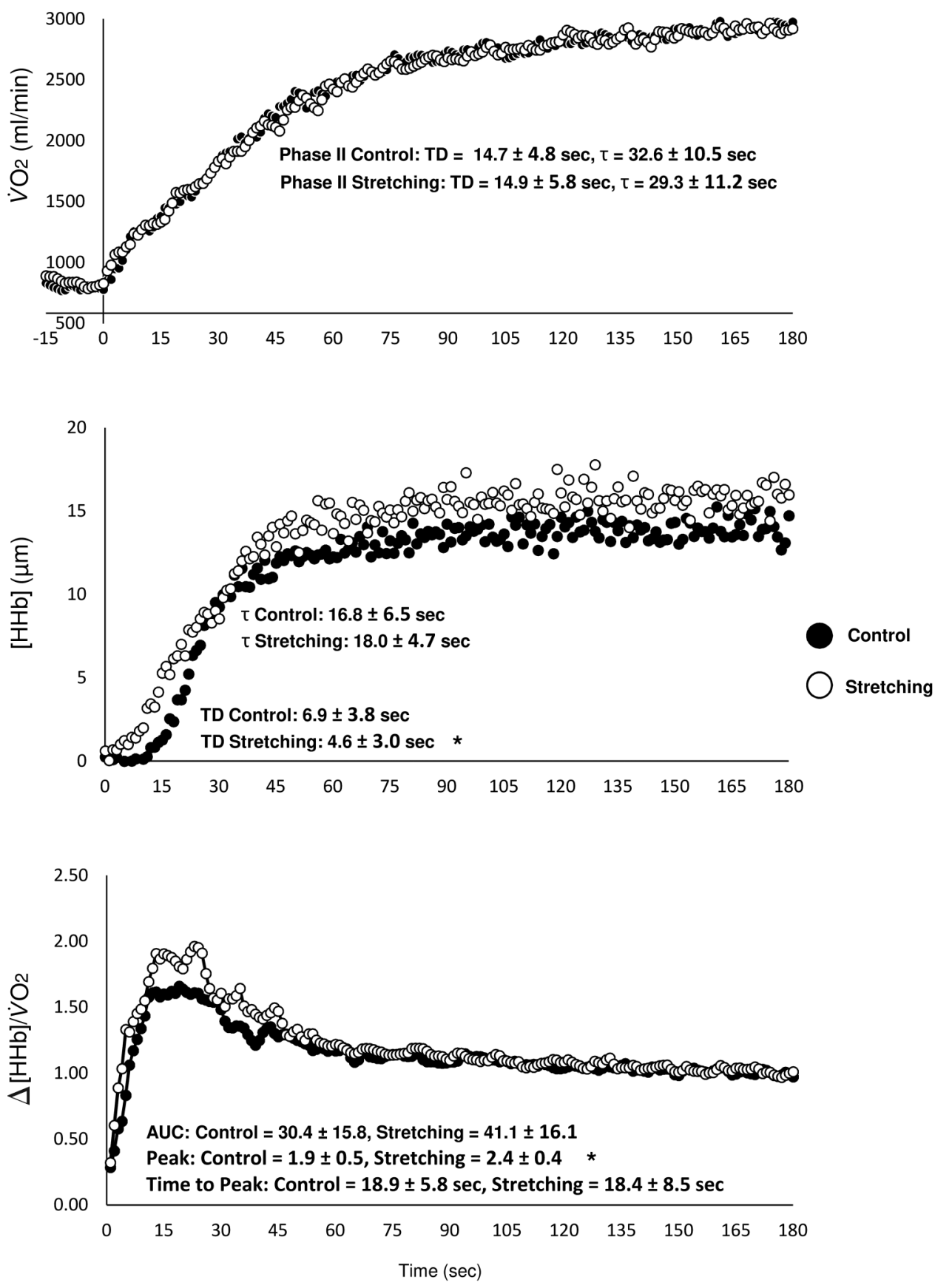

reduced by stretching, suggesting an influence of the increased muscle excitation on the kinetics of the slow component of $\mathrm{V}_{2}$. Finally, stretching was associated with an increased metabolic instability/mismatch between $\mathrm{O}_{2}$ delivery and $\mathrm{O}_{2}$ utilization during the on-kinetic, an increased perception of effort, and an increased $\left[\mathrm{La}^{-}\right]$. These phenomena are compatible with an increased contribution of the glycolytic energy system to sustain the same absolute intensity.

\section{Conflict of interest statement}

Authors declare no conflict of interest.

\section{Acknowledgements}

The authors express their gratitude to the subjects who made this data collection possible.

\section{References}

American College of Sports Medicine. 2017. ACSM'S Guidelines for Exercise Testing and Prescription, 10th ed. Edited by D. Riebe, J.K. Ehrman, G. Liguori, and M. Magal. Lippincott Williams \& Wilkins, Wolters Kluwer Health, Philadelphia, Pa., USA.

Bakeman, R. 2005. Recommended effect size statistics for repeated measures designs. Behav. Res. Methods, 37(3): 379-384. doi:10.3758/BF03192707. PMID: 16405133. 
Beaver, W.L., Wasserman, K., and Whipp, B.J. 1986. A new method for detecting anaerobic threshold by gas exchange. J. Appl. Physiol. 60(6): 2020-2027. doi: 10.1152/jappl.1986.60.6.2020. PMID:3087938.

Behm, D.G., Blazevich, A.J., Kay, A.D., and McHugh, M. 2016. Acute effects of muscle stretching on physical performance, range of motion, and injury incidence in healthy active individuals: a systematic review. Appl. Physiol. Nutr. Metab. 41(1): 1-11. doi:10.1139/apnm-2015-0235. PMID:26642915.

Borg, E., and Kaijser, L. 2006. A comparison between three rating scales for perceived exertion and two different work tests. Scand. J. Med. Sci. Sports, 16(1): 57-69. doi:10.1111/j.1600-0838.2005.00448.x. PMID:16430682.

Cannon, D.T., White, A.C., Andriano, M.F., Kolkhorst, F.W., and Rossiter, H.B. 2011. Skeletal muscle fatigue precedes the slow component of oxygen uptake kinetics during exercise in humans. J. Physiol. 589(3): 727-739. doi:10.1113/ jphysiol.2010.197723. PMID:21135050.

Colosio, A.L., Baldessari, E., Basso, E., and Pogliaghi, S. 2019. Respiratory and muscular response to acute non-metabolic fatigue during ramp incremental cycling. Respir. Physiol. Neurobiol. 270: 103281. doi:10.1016/j.resp.2019. 103281. PMID:31425884.

Cumming, G. 2014. The new statistics: why and how. Psychol. Sci. 25(1): 7-29. doi:10.1177/0956797613504966. PMID:24220629.

De Roia, G., Pogliaghi, S., Adami, A., Papadopoulou, C., and Capelli, C. 2012. Effects of priming exercise on the speed of adjustment of muscle oxidative metabolism at the onset of moderate-intensity step transitions in older adults. Am. J. Physiol. Regul. Integr. Comp. Physiol. 302(10): R1158-R1166. doi:10.1152/ajpregu.00269.2011. PMID:22422668.

Deley, G., Millet, G.Y., Borrani, F., Lattier, G., and Brondel, L. 2006. Effects of two types of fatigue on the VO2 slow component. Int. J. Sports Med. 27(6): 475482. doi:10.1055/s-2005-865837. PMID:16767612.

Esposito, F., Cè, E., and Limonta, E. 2012. Cycling efficiency and time to exhaustion are reduced after acute passive stretching administration. Scand. J. Med. Sci. Sports, 22: 737-745. doi:10.1111/j.1600-0838.2011.01327.x. PMID:21564308.

Ferreira, L.F., McDonough, P., Behnke, B.J., Musch, T.I., and Poole, D.C. 2006. Blood flow and $\mathrm{O} 2$ extraction as a function of $\mathrm{O} 2$ uptake in muscles composed of different fiber types. Respir. Physiol. Neurobiol. 153(3): 237-249. doi:10. 1016/j.resp.2005.11.004. PMID:16376620.

Field, A., Miles, J., and Field, Z. 2012. Discovering statistics using R. In SAGE, 1st edition. SAGE Publications Ltd, London. doi:10.5860/choice.50-2114.

Fontana, F.Y., Keir, D.A., Bellotti, C., De Roia, G.F., Murias, J.M., and Pogliaghi, S. 2015. Determination of respiratory point compensation in healthy adults: Can non-invasive near-infrared spectroscopy help? J. Sci. Med. Sport, 18(5): 590-595. doi:10.1016/j.jsams.2014.07.016. PMID:25153251.

Grassi, B., Pogliaghi, S., Rampichini, S., Quaresima, V., Ferrari, M., Marconi, C., and Cerretelli, P. 2003. Muscle oxygenation and pulmonary gas exchange kinetics during cycling exercise on-transitions in humans. J. Appl. Physiol. 95(1): 149-158. doi:10.1152/japplphysiol.00695.2002. PMID:12611769.

Grassi, B., Rossiter, H.B., and Zoladz, J.A. 2015. Skeletal muscle fatigue and decreased efficiency: two sides of the same coin? Exerc. Sport Sci. Rev. (2): 75-83. doi:10.1249/JES.0000000000000043. PMID:25688762.

Henneman, E., Somjen, G., and Carpenter, D.O. 1965. Functional significance of cell size in spinal motoneurons. J. Neurophysiol. 28(3): 560-580. doi:10.1152/ jn.1965.28.3.560. PMID:14328454.

Hermens, H.J., Freriks, B., Disselhorst-Klug, C., and Rau, G. 2000. Development of recommendations for SEMG sensors and sensor placement procedures. J. Electromyogr. Kinesiol. 10: 361-374. doi:10.1016/S1050-6411(00)00027-4. PMID:11018445.

Hopker, J.G., Caporaso, G., Azzalin, A., Carpenter, R., and Marcora, S.M. 2016. Locomotor muscle fatigue does not alter oxygen uptake kinetics during highintensity exercise. Front. Physiol. 7: 463. doi:10.3389/fphys.2016.00463. PMID: 27790156

Jensen, F.B. 2004. Red blood cell pH, the Bohr effect, and other oxygenation- linked phenomena in blood O2 and CO2 transport. Acta Physiol. Scand. 182(3): 215-227. doi:10.1111/j.1365-201X.2004.01361.x. PMID:15491402.

Jones, A.M., Grassi, B., Christensen, P.M., Krustrup, P., Bangsbo, J., and Poole, D.C. 2011. Slow component of $\dot{\mathrm{VO}}_{2}$ kinetics: mechanistic bases and practical applications. Med. Sci. Sports Exerc. 43(11): 2046-2062. doi:10.1249/ MSS.0b013e31821fcfc1. PMID:21552162.

Keir, D.A., Fontana, F., Robertson, T.C., Murias, J.M., Paterson, D.H., Kowalchuk, J.M., and Pogliaghi, S. 2015. Exercise intensity thresholds: identifying the boundaries of sustainable performance. Med. Sci. Sports Exerc. 47(9): 1932-1940. doi:10.1249/MSS.0000000000000613. PMID:25606817.

Keir, D.A., Pogliaghi, S., and Murias, J.M. 2018. The respiratory compensation point and the deoxygenation break point are valid surrogates for critical power and maximum lactate steady state. Med. Sci. Sports Exerc. 50(11): 2375-2378. doi:10.1249/MSS.0000000000001698. PMID:30134366.

Koga, S., Rossiter, H.B., Heinonen, I., Musch, T.I., and Poole, D.C. 2014. Dynamic heterogeneity of exercising muscle blood flow and $\mathrm{O} 2$ utilization. Med. Sci. Sports Exerc. 46(5): 860-876. doi:10.1249/MSS.0000000000000178. PMID: 24091989 .

Limonta, E., Rampichini, S., Riboli, A., Venturelli, M., Cè, E., and Esposito, F. 2015. Influence of acute passive stretching on the oxygen uptake vs work rate slope during an incremental cycle test. Eur. J. Appl. Physiol. 115(12): 25832592. doi:10.1007/s00421-015-3273-y. PMID:26433598.

Murias, J.M., Spencer, M.D., Kowalchuk, J.M., and Paterson, D.H. 2011a. Influence of phase I duration on phase II $\mathrm{VO}_{2}$ kinetics parameter estimates in older and young adults. Am. J. Physiol. Regul. Integr. Comp. Physiol. 301: R218-R224. doi:10.1152/ajpregu.00060.2011. PMID:21490368.

Murias, J.M., Spencer, M.D., Kowalchuk, J.M., and Paterson, D.H. 2011b. Muscle deoxygenation to $\mathrm{VO}_{2}$ relationship differs in young subjects with varying $\tau \dot{\mathrm{VO}}{ }_{2}$. Eur. J. Appl. Physiol. 111: 3107-3118. doi:10.1007/s00421-011-1937-9. PMID:21461928.

Olejnik, S., and Algina, J. 2003. Generalized eta and omega squared statistics: measures of effect size for some common research designs. Psychol. Methods, 8(4): 434-447. doi:10.1037/1082-989X.8.4.434. PMID:14664681.

Pogliaghi, S., Bellotti, C., and Paterson, D.H. 2014. "Tailored" submaximal step test for $\mathrm{VO}_{2}$ max prediction in healthy older adults. J. Aging Phys. Act. 22(2): 261-268. doi:10.1123/japa.2012-0171. PMID:23752341.

Poole, D., and Jones, A.M. 2012. Oxygen uptake kinetics. Compr. Physiol. 2(2): 933-996. doi:10.1002/cphy.c100072. PMID:23798293.

Tam, E., Bruseghini, P., Capelli, C., Oliboni, E., Pezzato, A., Pogliaghi, S., et al. 2018. Effect of endurance and strength training on the slow component of $\mathrm{O}_{2}$ kinetics in elderly humans. Front. Physiol. 9: 1353. doi:10.3389/fphys.2018. 01353. PMID:30356819.

Trajano, G.S., Nosaka, K., Seitz, L.B., and Blazevich, A.J. 2014. Intermittent stretch reduces force and central drive more than continuous stretch. Med. Sci. Sports Exerc. 46(5): 902-910. doi:10.1249/MSS.0000000000000185. PMID: 24121249.

Trajano, G.S., Nosaka, K., and Blazevich, A.J. 2017. Neurophysiological mechanisms underpinning stretch-induced force loss. Sports Med. 47(8): 1531-1541. doi:10.1007/s40279-017-0682-6. PMID:28120238.

Venturelli, M., Rampichini, S., Coratella, G., Limonta, E., Bisconti, A.V., Cé, E., and Esposito, F. 2019. Heart and musculoskeletal hemodynamic responses to repetitive bouts of quadriceps static stretching. J. Appl. Physiol. 127(2): 376384. doi:10.1152/japplphysiol.00823.2018. PMID:31161884.

Vigotsky, A.D., Halperin, I., Lehman, G.J., Trajano, G.S., and Vieira, T.M. 2018. Interpreting signal amplitudes in surface electromyography studies in sport and rehabilitation sciences. Front. Physiol. 8: 985. doi:10.3389/fphys.2017. 00985. PMID:29354060.

Whipp, B.J., Davis, J.A., and Wasserman, K. 1989. Ventilatory control of the "isocapnic buffering" region in rapidly-incremental exercise. Respir. Physiol. 76(3): 357-367. doi:10.1016/0034-5687(89)90076-5. PMID:2501844. 